\title{
All-optical combiner-splitter and gating devices based on straight waveguides
}

\author{
Matthew Wescott, ${ }^{1}$ Guangzhou Chen, ${ }^{2}$ Yanyun Zhang, ${ }^{2}$ Brian G. Bagley, ${ }^{2}$ and Robert T. Deck ${ }^{2, *}$ \\ ${ }^{1}$ Department of Physics, California Institute of Technology, Pasadena, California 91126 \\ 2Department of Physics and Astronomy, University of Toledo, Toledo, Ohio 43606 \\ ${ }^{*}$ Corresponding author: rtd@physics.utoledo.edu
}

Received 24 April 2006; revised 12 September 2006; accepted 18 September 2006; posted 30 January 2007 (Doc. ID 70202); published 15 May 2007

\begin{abstract}
A two-mode optical combiner-splitter device is designed based on all straight waveguides that maintains the integrity of the two modes during propagation and allows for an analytic analysis. The design analysis has the potential to improve the precision of the device fabrication. The design is used in an analytic optical gate based on a nonlinear Mach-Zehnder interferometer. The design reduces the size of a previously proposed device and simplifies its analysis. (C) 2007 Optical Society of America

OCIS codes: $\quad 230.1150,230.3120,230.7390,230.4320$.
\end{abstract}

\section{Introduction}

The need for rapid processing of high bit-rate optical signals has stimulated interest in all-optical waveguide devices of increasing functional complexity. These devices can be most simply fabricated in planar geometry. Recently, for example, an all-optical transistor has been proposed by Medhekar and Sarkar [1]. In this device a $\mathrm{Y}$ combiner directs control (gate) and signal beams into a single-mode waveguide providing the input to a nonlinear optical device, which serves to modulate the output signal [2]. It is an advantage of the device that a relatively weak control beam effects a change in an intense beam (as in an electronic transistor). On the other hand, it is a disadvantage of the device that the control beam is not extracted from the signal beam at the output, and spurious effects producing small phase shifts in either (or both) of the two input beams prior to the Y combiner can also produce changes in the output. These effects, either singly or together, can produce significant control problems in the case in which the optical transistors are sequentially cascaded in the manner of electronic transistors. Also recently, an all-optical logic gate has been proposed by Yabu et al. [3] consisting of a modified MachZehnder interferometer with a nonlinear section in one

0003-6935/07/163177-08\$15.00/0

(C) 2007 Optical Society of America arm (analogous to a commercial electro-optic MachZehnder modulator). The gating properties of this device were determined by simulation. We describe this device further in Section 3.

In this paper we propose a planar geometry element for combining and separating optical signals, which can be analyzed entirely analytically, so as to simplify both its fabrication and design. Specifically, we replace the curved entrance and exit channels of the usual Y splitters and combiners used in Refs. [1] and [3] with straight waveguides of different widths, designed to couple to only one of the distinct modes of a two-mode channel supporting both a symmetric and an antisymmetric mode. The resulting device allows two signals of the same (or different) wavelength to be combined into a single channel and subsequently separated after an arbitrary propagation length, without change in either signal. Its use at the entrance and exit ends of the optical device in Refs. [1] and [2] would allow for the separation of the output control and signal beams.

As photonic integrated circuits become more complex, and the tolerances on their fabrication become more restrictive, we anticipate that the need for design precision will increase, and the analytically tractable design we propose here will find use in different applications. We provide the details of our design in Section 2 . As an example, we then apply our design to the all-optical logic gate introduced in Ref. [3]. The required analysis is summarized in Section 3, and 
the results obtained in this case for a given set of parameters are discussed in Section 4.

\section{Details of Proposed Design}

Figure 1 illustrates the general layout of our design, in which power in the symmetric mode of a multimode channel $\mathrm{D}$ is transferred into and out of channel $\mathrm{D}$ through single-mode channels $\mathrm{C}$ and $\mathrm{F}$, and power in the antisymmetric mode of Channel $\mathrm{D}$ is transferred into and out of channel $D$ through single-mode channels $B_{1}$ and $B_{2}$. To effect these power transfers, for use as a combiner or divider, the propagation constant of the symmetric single mode of channels $B_{1}$ and $\mathrm{B}_{2}$ is designed to equal the propagation constant of the antisymmetric mode of channel $\mathrm{D}$, so as to allow power in the symmetric mode of channel $B_{1}$ to transfer into the antisymmetric mode of channel $\mathrm{D}$ with a coupling length $\mathrm{L}$, and power in the antisymmetric mode of channel $\mathrm{D}$ to transfer into channel $\mathrm{B}_{2}$ with the same coupling length. The coupling between channels $B_{1}$ and $C$, or $B_{2}$ and $F$, is minimized by the distinct propagation constants of the modes of these pairs of channels. Region III, depending on the application, can be either a linear or nonlinear optical waveguide, as determined by the input power and waveguide composition.

Figure 2 presents the result of a numerical evaluation of the field intensity versus propagation distance $z$ in the geometry of Fig. 1 for the case in which a field initiates in the channel of width $w_{1}$. The displayed dependence of the intensity on $z$ shows that the symmetric mode of the right-hand channel $\mathrm{B}_{1}$ transfers in a coupling length $\mathrm{L}=550 \mu \mathrm{m}$ exclusively into the

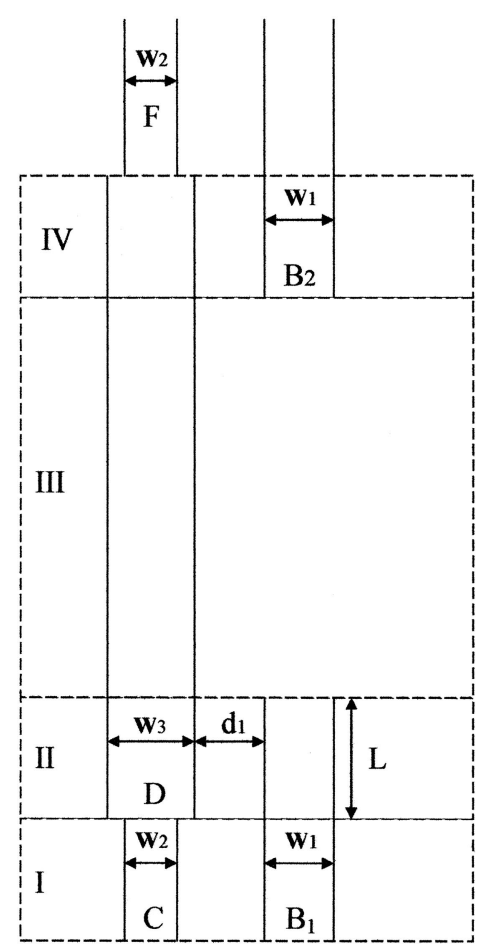

Fig. 1. Diagram of the signal combiner/splitter device based on straight waveguides. antisymmetric mode of the left-hand channel $\mathrm{D}$, which subsequently transfers over the same coupling length into the symmetric mode of the output channel on the right. The parameters used to effect the result are listed in the caption of Fig. 2. To assess fabrication tolerances, we have altered the length $\mathrm{L}$ along channel $\mathrm{B}_{1}$ in comparison to the computed coupling length of $550 \mu \mathrm{m}$. Our results indicate that a change in L of $\pm 20 \mu \mathrm{m}$ (which greatly exaggerates the expected fabrication errors) changes the power transferred to channel $\mathrm{D}$ in Fig. 1 by less than $0.5 \%$, so as to make only a negligible change in the graph in Fig. 2.

\section{Theory Related to Application}

Here we apply the above design to an alternative version of the gating device proposed in Ref. [3]. In the original device, shown schematically in Fig. 3, the nonlinear section $N$ is designed to change the phase of a signal by $\pi$ in the presence of a second signal injected into a control channel $\mathrm{C}$ before the nonlinear section and removed before the output of the device. To allow the removal of the control beam, the arm of the interferometer having the nonlinear section must support two modes, and the coupling to the two modes must be independently controllable. To accomplish this, the input and output channels are single mode, and their widths are chosen to allow coupling to only a single mode of the multimode section [4]. Y splitter-combiner configurations are used at the input and output of the gate. It is a significant result of Ref. [3] that the function of the device is insensitive to the relative phase difference between the signal and control beams.

Figure 4 presents an overview of our alternative design for the logic gate. The proposed device is an interferometer with left and right arms consisting of a sequence of parallel waveguides, each of which carries a mode that couples exclusively to a single mode in a neighboring channel [5]. In the operation of the device, evanescent coupling between channels $B_{1}, B_{1}{ }^{\prime}$ and $\mathrm{A}$ in Region I causes an input signal in channel $\mathrm{A}$ to divide into equal signals in channels $B_{1}$ and $B_{1}{ }^{\prime}$, which subsequently combine in or out of phase in channel X. Before the signals recombine, one of them passes through a nonlinear section $\mathrm{N}$ in which the final phase of the signal is controlled by a second beam incident in channel $\mathrm{C}$. The loss in the device is minimized by terminating the channels at lengths for which the powers in the terminated channels are minimized [5].

It is useful to emphasize the role of the separate regions in the design of Fig. 4. In Region 0, signal and control beams enter uncoupled single-mode channels $\mathrm{A}$ and $\mathrm{C}$ supporting distinct modes characterized by distinct propagation constants. In Region I, channel A passes between equidistant channels $B_{1}$ and $B_{1}{ }^{\prime}$ for a distance $\mathrm{L}_{\mathrm{I}}$, after which the power in channel $A$ has transferred completely and symmetrically into the parallel channels $\mathrm{B}_{1}$ and $\mathrm{B}_{1}{ }^{\prime}$. The coupling between the modes in channels $B_{1}$ and $C$ is eliminated by the designed mismatch of their propagation constants. In Region II the single-mode channel $\mathrm{C}$ joins to a two- 


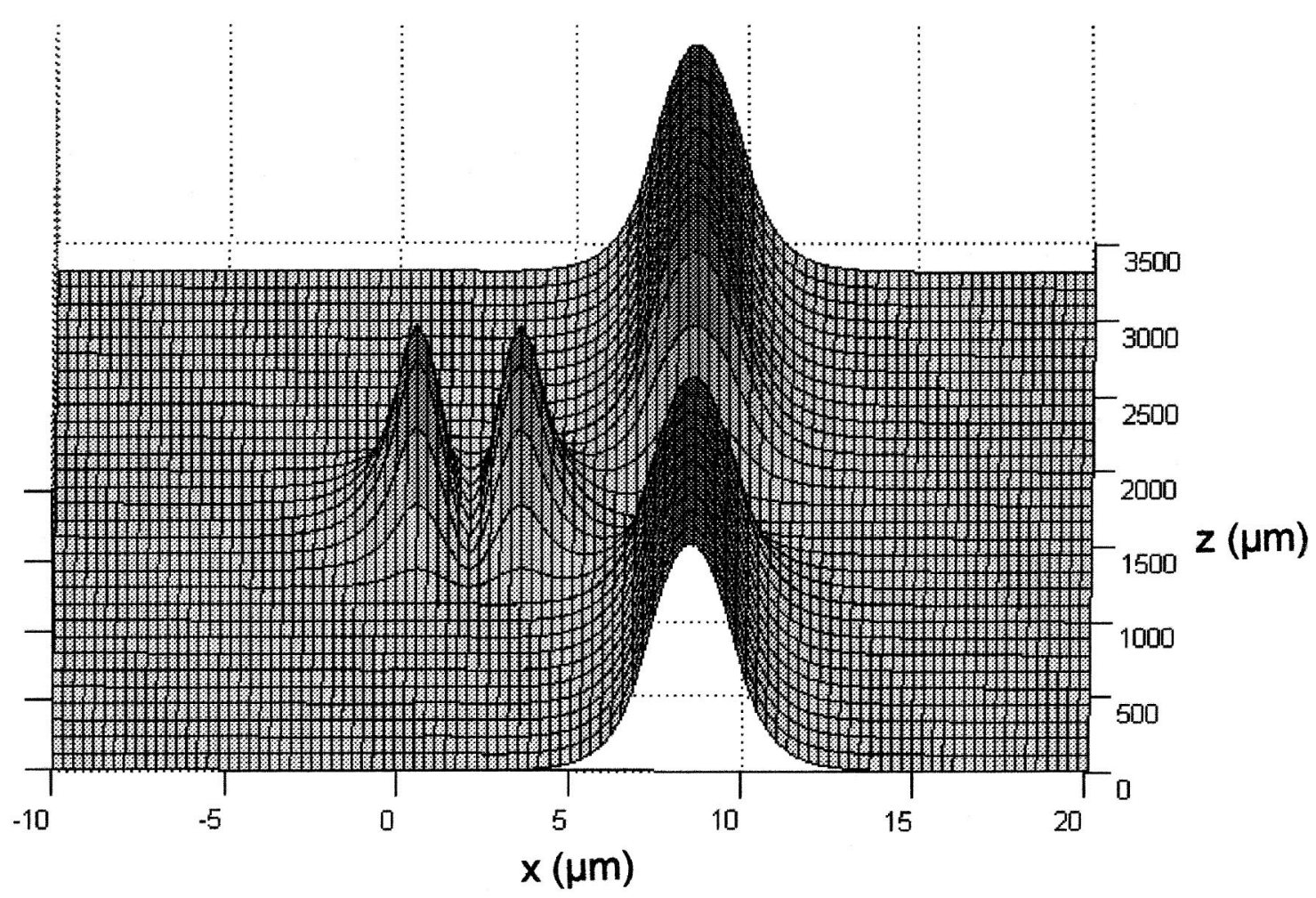

Fig. 2. Numerical evaluation of the field intensity versus $z$ in channels of widths $w_{1}$ and $w_{3}$, for the case that the field initiates in the channel of width $w_{1}$. Designed parameter values in Fig. 1 are: $\lambda=1.55 \mu \mathrm{m}, w_{1}=3.0 \mu \mathrm{m}, w_{3}=4.0 \mu \mathrm{m}, d_{1}=3.0 \mu \mathrm{m}$, index of refraction of cladding, $n_{\mathrm{o}}=1.444$, index of channels $B_{1}$ and $B_{2}, n_{1}=1.4589$, index of channels $C$ and $D, n_{2}=n_{3}=1.4754$. Plot shows transfer of symmetric mode in right-hand channel into and out of antisymmetric mode in left-hand channel.

mode channel D supporting a symmetric and an antisymmetric mode, while channels $\mathrm{B}_{1}$ and $\mathrm{B}_{1}{ }^{\prime}$ are uncoupled and separately coupled to channels $\mathrm{D}$ and

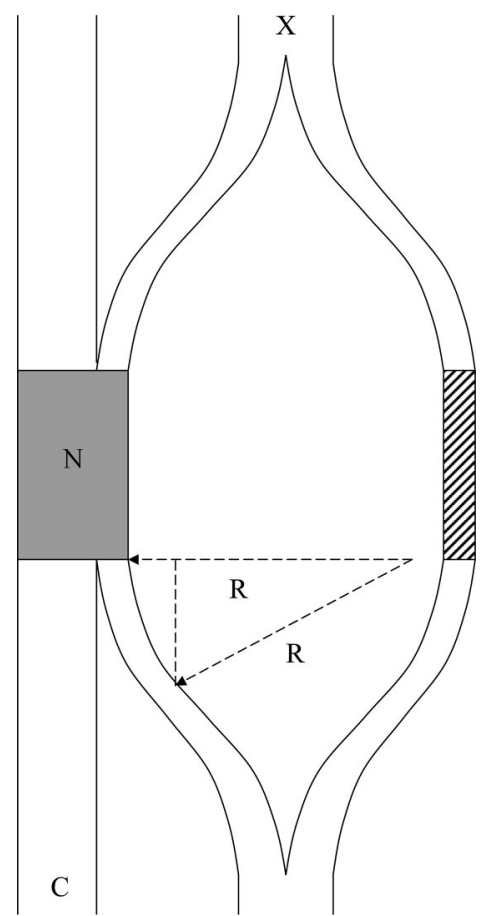

Fig. 3. Diagram of the optical gating device designed in Ref. 3.
$\mathrm{D}^{\prime}$, respectively. The propagation constants of the symmetric single mode of channel $\mathrm{B}_{1}$ and the antisymmetric mode of channel $\mathrm{D}$ are designed to be equal, to allow the power in channel $\mathrm{B}_{1}$ to transfer into the antisymmetric mode of channel $\mathrm{D}$ with a coupling length $\mathrm{L}_{\mathrm{II}}$. The power in channel $\mathrm{D}$ at the terminus of region II is the sum of the powers in the symmetric mode initiated in channel $\mathrm{C}$ and the antisymmetric mode transferred into channel D from channel A. In Region III channel D joins to a two-mode nonlinear channel $\mathrm{N}$ designed so that the simultaneous presence of the symmetric and antisymmetric modes in the channel shifts the phase of the antisymmetric mode by $\pi$ (relative to the phase of the antisymmetric mode in channel $\mathrm{D}^{\prime}$ ) during a length $\mathrm{L}_{\mathrm{III}}$. In Region IV channel $\mathrm{N}$ joins to channel $\mathrm{E}$, which supports the symmetric and antisymmetric modes of channel $\mathrm{N}$, and channel $\mathrm{B}_{2}$ initiates parallel to channel E. The latter channel terminates at a single-mode channel $\mathrm{F}$ after a distance $\mathrm{L}_{\mathrm{IV}}$, at which the antisymmetric mode of channel $\mathrm{N}$ has transferred into channel $\mathrm{B}_{2}$, and the symmetric mode of channel $\mathrm{N}$ has transferred into channel $\mathrm{F}$. The right arm of the device operates in a similar way. In Region $\mathrm{V}$ the signals in channels $\mathrm{B}_{2}$ and $\mathrm{B}_{2}{ }^{\prime}$ transfer completely into the output channel $X$ after a coupling length $L_{V}$, resulting in an output signal either identical to the incident signal in channel A, or null, depending on the state of the control beam in channel C. Incidental coupling between the modes of channels $\mathrm{B}_{2}$ and $\mathrm{F}$ and 


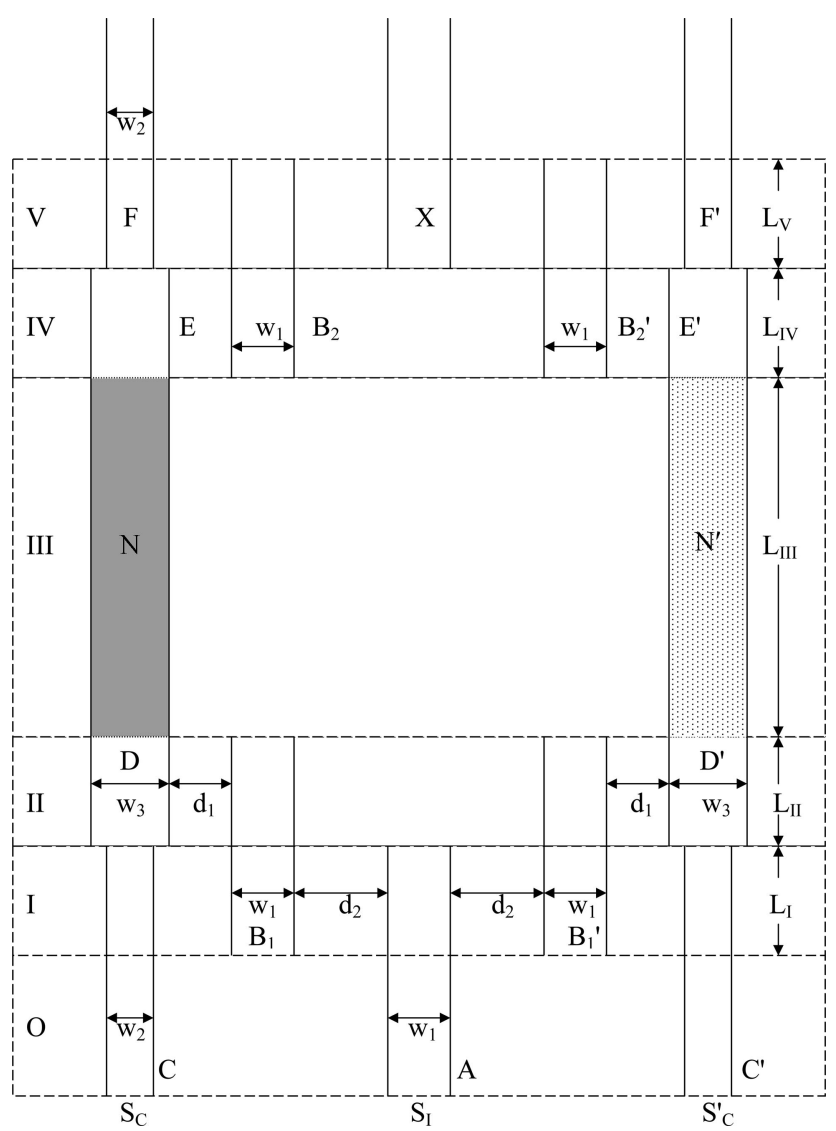

Fig. 4. Diagram of optical gate proposed here. Lengths along the transverse $x$ direction are expanded by a factor of 100 compared with lengths along the $z$ direction. The index of refraction is $n_{1}$ in channels $A, B_{1}, B_{2}, B_{1}{ }^{\prime}, B_{2}{ }^{\prime}$, and $X, n_{2}$ in channels $C, D, E, F, C^{\prime}, D^{\prime}$, $E^{\prime}, F^{\prime}$, and $\sqrt{n_{3}{ }^{2}+\alpha|E|^{2}}$ in channels $N$ and $N^{\prime}$. The index of the channel claddings is $n_{0}$.

the modes of channels $\mathrm{B}_{2}{ }^{\prime}$ and $\mathrm{F}^{\prime}$ is eliminated by the mismatch between their propagation constants.

An advantage of the proposed device is its bilateral symmetry, which allows it to be cascaded with similar elements in a logic circuit (in forward or reverse mode) and guarantees the two equalities $\mathrm{L}_{\mathrm{IV}}=\mathrm{L}_{\mathrm{II}}$ and $\mathrm{L}_{\mathrm{V}}=\mathrm{L}_{\mathrm{I}}$.

A determination of the parameters needed for the devices in Figs. 1, 3, and 4 requires a calculation of the profiles and phases of the fields within the waveguide channels. By eliminating the curved channels at the ends of the device in Fig. 3, an analytic evaluation of the modes of the device in Fig. 4 is made possible. This allows the field in the latter device to be represented as a linear combination of the supermodes of the coupled channels. For comparison with the simulation results of Yabu et al. [3], it is sufficient to reduce the analysis of the device in Fig. 4 to the two dimensions labeled $x$ and $z$ by use of the slab approximation. The simplification models the proposed gating device as a dielectric structure defined by a dielectric function, $\varepsilon(x, z)$, with a dependence on the transverse coordinate $x$ reflecting the discrete changes in index at the boundaries of the guiding channels. The spatial dependence of an electromag- netic field of angular frequency $\omega$ in the $N$ th region of the device is then well described by an equation expressible in Gaussian units as

$$
\left[\frac{\partial^{2}}{\partial x^{2}}+\frac{\partial^{2}}{\partial z^{2}}+\frac{\omega^{2}}{c^{2}} \varepsilon_{N}(x, z)\right] \mathrm{E}_{N}(x, z)=0
$$

where $\mathrm{E}_{N}(x, z)$ is the electric field amplitude in region $N,(N=0, \mathrm{I}, \mathrm{II}, \mathrm{III}, \mathrm{IV}, \mathrm{V})$, and $\varepsilon_{N}$ is dependent on $z$ only in the nonlinear region of the device (III). Below we take $\varepsilon_{N}$ in the nonlinear region to have a Kerr-type quadratic dependence on the electric field expressible as

$$
\varepsilon_{\mathrm{III}}(x, z)=\varepsilon_{\mathrm{III}}(x)+\alpha\left|\mathrm{E}_{\mathrm{III}}(x, z)\right|^{2},
$$

where $\varepsilon_{\text {III }}(x)$ denotes the linear dielectric constant in the limit of zero field, and $\alpha$ is a constant, equated here to the value $1.8 \times 10^{-16} \mathrm{~m}^{2} / \mathrm{V}^{2}$ adopted in Ref. [3]. The dependence of the dielectric constant on the field intensity in the nonlinear medium results in a periodic exchange of power between the modes of the nonlinear region with a period determined by $\alpha$ [6].

An evaluation of the linear modes requires a satisfaction of the polarization-dependent continuity conditions at the boundaries of the channels. For this purpose we assume the electric vector of the fields to be polarized along the $y$ axis (as in Ref. [3]).

Neglecting radiation losses, the solution of Eq. (1) corresponding to a guided field propagating in the $z$ direction in Region $\mathrm{N}$ of the structure, can be expressed as a linear combination of the (linear) waveguide modes of the region in the form

$$
\mathrm{E}_{N}(x, z)=\sum_{j} a_{N j}(z)^{\mathscr{E}} \mathscr{E}_{N j}(x) \exp \left(\mathrm{i} \beta_{N j} z\right)
$$

where $\varepsilon_{N j}(x)$ and $\beta_{N j}$ represent, respectively, the transverse profile function and propagation constant of the $j$ th supermode of the region, defined by the equation

$$
\left[\frac{\mathrm{d}^{2}}{\mathrm{~d} x^{2}}+\frac{\omega^{2}}{c^{2}} \varepsilon_{N}(x)-\beta_{N j}\right] \mathscr{E}_{N j}(x)=0 .
$$

In what follows we take the (real) profile functions $\varepsilon_{j}(x)$ to satisfy the orthonormality relation

$$
\int_{-\infty}^{\infty} \mathrm{d} x \mathscr{E}_{N j}(x)^{\mathscr{E}_{N j^{\prime}}}(x)=\delta_{j j^{\prime}} .
$$

In the linear regions of the gate, in which the coefficients $a_{N j}$ are independent of $z$, relation (5) makes it possible to determine the coefficients from the value of the field at the entrance end of the region, at $z=z_{N_{0}}$, by multiplication of Eq. (3) by $\mathscr{E}_{N j^{\prime}}(x)$ and integration over $x$ with the help of Eq. (5) to obtain the formula 
$a_{N j}\left(z_{N o}\right)=\int_{-\infty}^{\infty} \mathrm{d} x \mathscr{E}_{N j}(x) \mathrm{E}\left(x, z_{N o}\right) \exp \left(-\mathrm{i} \beta_{N j} z_{N o}\right)$.

A determination of the field in the nonlinear region of structure (III) requires an evaluation of the $z$-dependent coefficients $a_{\mathrm{IIJj}}(z)$ by solution of Eq. (1) with $\varepsilon_{\mathrm{III}}(x, z)$ in the form of Eq. (2). Substituting into Eq. (2) the representation for $\mathrm{E}_{\mathrm{III}}(x, z)$ in Eq. (3), reexpresses $\varepsilon_{\mathrm{III}}(x, z)$ as

$\begin{aligned} \varepsilon_{\mathrm{III}}(x, z)= & \varepsilon_{\mathrm{III}}(x)+\alpha\left\{\left|a_{1}(z)\right|^{2}\left|\mathscr{E}_{1}(x)\right|^{2}+\left|a_{2}(z)\right|^{2}\left|\mathscr{E}_{2}(x)\right|^{2}\right. \\ & \left.+2 \operatorname{Re} a_{1}(z) a_{2}{ }^{*}(z)^{\mathscr{E}}(x) \mathscr{E}_{2}(x) \exp \left[\mathrm{i}\left(\beta_{1}-\beta_{2}\right) z\right]\right\},\end{aligned}$

where the subscripts 1 and 2 label the two (symmetric and antisymmetric) modes of the nonlinear region respectively, and the subscript III on $a_{j}, \mathscr{E}_{j}$, and $\beta_{j}$ is suppressed for simplicity. The insertion into Eq. (1) of the forms for $\varepsilon_{\mathrm{III}}(x, z)$ and $\mathrm{E}_{\mathrm{III}}(x, z)$ in Eqs. (2) and (3), and the use of Eq. (4) results in the equation

$$
\begin{gathered}
\mathscr{E}_{1} \mathrm{e}^{\mathrm{i} \beta_{1} z}\left[\frac{\mathrm{d}^{2} a_{1}}{\mathrm{~d} z^{2}}+2 \mathrm{i} \beta_{1} \frac{\mathrm{d} a_{1}}{\mathrm{~d} z}\right]+\mathscr{E}_{2} \mathrm{e}^{\mathrm{i} \beta_{2} z}\left[\frac{\mathrm{d}^{2} a_{2}}{\mathrm{~d} z^{2}}+2 \mathrm{i} \beta_{2} \frac{\mathrm{d} a_{2}}{\mathrm{~d} z}\right] \\
+\frac{\omega^{2}}{c^{2}} \alpha\left[a_{1} \mathscr{E}_{1} \mathrm{e}^{\mathrm{i} \beta_{1} z}+a_{2} \mathscr{E}_{2} \mathrm{e}^{\mathrm{i} \beta_{2} z}\right]\left|\mathrm{E}_{\mathrm{III}}(x, z)\right|^{2}=0
\end{gathered}
$$

The equation can be simplified by the assumption that the mode amplitudes vary slowly in space on the length scales determined by the reciprocals of the mode propagation constants $\beta_{j}$, consistent with the relations

$$
\frac{\mathrm{d} a_{j}(z)}{\mathrm{d} z} \ll \beta_{j} a_{j}(z), \quad \frac{\mathrm{d}^{2} a_{j}(z)}{\mathrm{d} z^{2}} \ll \beta_{j} \frac{\mathrm{d} a_{j}(z)}{\mathrm{d} z},
$$

which permit the neglect of the second derivative terms in Eq. (8) compared with the first derivative terms, to reduce the equation to the form

$$
\begin{aligned}
& 2 \mathrm{i} \beta_{1} \mathscr{E}_{1}(x) \mathrm{e}^{\mathrm{i} \beta_{1} z} \frac{\mathrm{d} a_{1}}{\mathrm{~d} z}+2 \mathrm{i} \beta_{2} \mathscr{E}_{2}(x) \mathrm{e}^{\mathrm{i} \beta_{2} z} \frac{\mathrm{d} a_{2}}{\mathrm{~d} z}+\frac{\omega^{2}}{c^{2}} \alpha\left[a_{1} \mathscr{E}_{1}(x) \mathrm{e}^{\mathrm{i} \beta_{1} z}\right. \\
& \left.+a_{2} \mathscr{E}_{2}(x) \mathrm{e}^{\mathrm{i} \beta_{2} z}\right]\left|\mathrm{E}_{\mathrm{III}}(x, z)\right|^{2}=0 .
\end{aligned}
$$

A separate multiplication of this equation by $\mathscr{E}_{1}(x) \mathrm{e}^{-\mathrm{i} \beta_{1} z}$ and $\mathscr{E}_{2}(x) \mathrm{e}^{-\mathrm{i} \beta_{2} z}$ and the integration of the resulting two equalities over all $x$ using Eq. (5) produces two coupled equations for the amplitudes $a_{j}(z)$ expressible as

$$
\frac{\mathrm{d} a_{1}(z)}{\mathrm{d} z}=\frac{\mathrm{i}}{\beta_{1}} \mathrm{Q}_{1}(z) a_{1}(z)+\frac{\mathrm{i}}{\beta_{1}} \mathrm{Q}_{12}(z) a_{2}(z) \mathrm{e}^{\mathrm{i}\left(\beta_{2}-\beta_{1}\right) z},
$$

$$
\frac{\mathrm{d} a_{2}(z)}{\mathrm{d} z}=\frac{\mathrm{i}}{\beta_{2}} \mathrm{Q}_{2}(z) a_{2}(z)+\frac{\mathrm{i}}{\beta_{2}} \mathrm{Q}_{12}(z) a_{1}(z) \mathrm{e}^{\mathrm{i}\left(\beta_{1}-\beta_{2}\right) z}
$$

where

$$
\begin{aligned}
\mathrm{Q}_{j}(z) & =\frac{\omega^{2}}{c^{2}} \frac{\alpha}{2} \int_{-\infty}^{\infty} \mathrm{d} x \mathscr{E}_{j}^{2}(x)\left|\mathrm{E}_{\mathrm{III}}(x, z)\right|^{2}, \\
\mathrm{Q}_{12}(z) & =\frac{\omega^{2}}{c^{2}} \frac{\alpha}{2} \int_{-\infty}^{\infty} \mathrm{d} x \mathscr{E}_{1}(x) \mathscr{E}_{2}(x)\left|\mathrm{E}_{\mathrm{III}}(x, z)\right|^{2} .
\end{aligned}
$$

By use of the form for $\mathrm{E}_{\mathrm{III}}(x, z)$ in Eq. (3), the $z$ dependence of the quantities $\mathrm{Q}_{j}$ and $\mathrm{Q}_{12}$ can be written more explicitly in the forms

$$
\begin{aligned}
\mathrm{Q}_{1}(z)= & \frac{\omega^{2}}{c^{2}} \frac{\alpha}{2}\left\{\mathrm{~K}_{1}\left|a_{1}(z)\right|^{2}+\mathrm{K}_{12}\left|a_{2}(z)\right|^{2}\right. \\
& \left.+2 \mathrm{~K}_{13} \operatorname{Re} a_{1}(z) a_{2}^{*}(z) \mathrm{e}^{\mathrm{i}\left(\beta_{1}-\beta_{2}\right) z}\right\}, \\
\mathrm{Q}_{2}(z)= & \frac{\omega^{2}}{c^{2}} \frac{\alpha}{2}\left\{\mathrm{~K}_{2}\left|a_{2}(z)\right|^{2}+\mathrm{K}_{12}\left|a_{1}(z)\right|^{2}\right. \\
& \left.+2 \mathrm{~K}_{23} \operatorname{Re} a_{1}(z) a_{2}^{*}(z) \mathrm{e}^{\mathrm{i}\left(\beta_{1}-\beta_{2}\right) z}\right\}, \\
\mathrm{Q}_{12}(z)= & \frac{\omega^{2}}{c^{2}} \frac{\alpha}{2}\left\{\mathrm{~K}_{13}\left|a_{1}(z)\right|^{2}+\mathrm{K}_{23}\left|a_{2}(z)\right|^{2}\right. \\
& \left.+2 \mathrm{~K}_{12} \operatorname{Re} a_{1}(z) a_{2}^{*}(z) \mathrm{e}^{\mathrm{i}\left(\beta_{1}-\beta_{2}\right) z}\right\},
\end{aligned}
$$

with the definitions

$$
\begin{aligned}
\mathrm{K}_{1} & \equiv \int_{-\infty}^{\infty} \mathrm{d} x \mathscr{E}_{1}{ }^{4}(x), \quad \mathrm{K}_{2} \equiv \int_{-\infty}^{\infty} \mathrm{d} x \mathscr{E}_{2}{ }^{4}(x), \\
\mathrm{K}_{12} & \equiv \int_{-\infty}^{\infty} \mathrm{d} x \mathscr{E}_{1}{ }^{2}(x)^{\mathscr{E}_{2}}{ }^{2}(x), \quad \mathrm{K}_{13} \equiv \int_{-\infty}^{\infty} \mathrm{d} x_{\mathscr{E}_{1}}{ }^{3}(x)^{\mathscr{E}} 2(x), \\
\mathrm{K}_{23} & \equiv \int_{-\infty}^{\infty} \mathrm{d} x \mathscr{E}_{1}(x)^{\mathscr{E}_{2}}{ }^{3}(x) .
\end{aligned}
$$

Here, because $\mathscr{E}_{1}(x)$ and $\mathscr{E}_{2}(x)$ are respectively symmetric and antisymmetric functions of $x$, the coefficients $\mathrm{K}_{13}$ and $\mathrm{K}_{23}$ are identically zero. The substitution of Eqs. (13a-c) into Eqs. (11), with $\mathrm{K}_{13}=\mathrm{K}_{23}=0$, then results in the more explicit equations for the amplitudes $a_{j}(z)$ :

$$
\begin{aligned}
\frac{\mathrm{d} a_{1}}{\mathrm{~d} z}= & \frac{\mathrm{i}}{\beta_{1}} \frac{\omega^{2}}{c^{2}} \frac{\alpha}{2}\left\{\mathrm{~K}_{1}\left|a_{1}\right|^{2} a_{1}+\mathrm{K}_{12}\left|a_{2}\right|^{2} a_{1}\right. \\
& \left.+2 \mathrm{~K}_{12} \operatorname{Re}\left[a_{1} a_{2}{ }^{*} \mathrm{e}^{\mathrm{i}\left(\beta_{1}-\beta_{2}\right) z}\right] a_{2} \mathrm{e}^{\mathrm{i}\left(\beta_{2}-\beta_{1}\right) z}\right\}, \\
\frac{\mathrm{d} a_{2}}{\mathrm{~d} z}= & \frac{\mathrm{i}}{\beta_{2}} \frac{\omega^{2}}{c^{2}} \frac{\alpha}{2}\left\{\mathrm{~K}_{2}\left|a_{2}\right|^{2} a_{2}+\mathrm{K}_{12}\left|a_{1}\right|^{2} a_{2}\right. \\
& \left.+2 \mathrm{~K}_{12} \operatorname{Re}\left[a_{1} a_{2}{ }^{*} \mathrm{e}^{\mathrm{i}\left(\beta_{1}-\beta_{2}\right) z}\right] a_{1} \mathrm{e}^{\mathrm{i}\left(\beta_{1}-\beta_{2}\right) z}\right\} .
\end{aligned}
$$


Of particular significance are the phases of the coefficients $a_{j}\left(=a_{\mathrm{IIIj}}\right)$, which Eqs. (15) define to be complex functions of $z$. It is useful to represent these coefficients in the form

$$
a_{j}(z)=a_{j}\left(z_{\mathrm{III}}\right) \rho_{j}(z) \exp \left[\mathrm{i} \theta_{j}(z)\right],
$$

where $a_{j}\left(z_{\mathrm{III}}\right)$ is the value of $a_{j}(z)$ at the entrance to the nonlinear region, and $\rho_{j}(z)$ and $\theta_{j}(z)$ denote the corrections to the amplitude and phase of $a_{j}\left(z_{\mathrm{III}}\right)$ produced by the nonlinearity. The use of the Euler relation connects the phase $\theta_{j}(z)$ to the real and imaginary parts of $a_{j}(z)$ through the equation

$$
\theta_{j}(z)=\tan ^{-1}\left[\operatorname{Im} a_{j}(z) / \operatorname{Re} a_{j}(z)\right] .
$$

The substitution of the form for $a_{j}(z)$ in Eq. (16) into Eq. (3), expresses the field at an arbitrary value of $z$ in the nonlinear medium in the form

$$
\begin{aligned}
\mathrm{E}_{\mathrm{III}}(x, z)= & {\left[a_{1}\left(z_{\mathrm{IIIo}}\right) \rho_{1}(z) \exp \left(\mathrm{i}\left[\beta_{1} z+\theta_{1}(z)\right]\right) \varepsilon_{1}(x)\right.} \\
& \left.+a_{2}\left(z_{\mathrm{IIIo}}\right) \rho_{2}(z) \exp \left(\mathrm{i}\left[\beta_{2} z+\theta_{2}(z)\right]\right) \varepsilon_{2}(x)\right],
\end{aligned}
$$

consistent with a $z$-dependent phase difference between the symmetric and antisymmetric modes of the nonlinear channel given by

$$
\left(\beta_{1}-\beta_{2}\right) z+\left[\theta_{1}(z)-\theta_{2}(z)\right] .
$$

\section{Results and Conclusions}

The design of the devices in Figs. 1 and 4 requires a choice of values for the dielectric constants and the parameters $w_{1}, w_{3}$, and $d_{1}$ resulting in a match of the propagation constant of the symmetric mode of channels $B_{1}$ and $B_{2}$ to the propagation constant of the antisymmetric mode of channels $\mathrm{D}$ and $\mathrm{E}$; so as to result in a transfer of the field in channel $B_{1}$ exclusively into the antisymmetric mode of channel $\mathrm{D}$, and subsequent transfer of the antisymmetric mode of channel $\mathrm{E}$ into channel $\mathrm{B}_{2}$. For a given choice of wavelength $\lambda$, a suitable set of parameters for this purpose can be extracted from the dispersion relation for the modes of a slab waveguide of width $w$, expressible as

$$
w \sqrt{\left(\frac{2 \pi}{\lambda}\right)^{2} \varepsilon_{\mathrm{g}}-\beta^{2}}=2 \tan ^{-1}\left(\frac{\sqrt{\beta^{2}-\left(\frac{2 \pi}{\lambda}\right)^{2} \varepsilon_{\mathrm{c}}}}{\sqrt{\left(\frac{2 \pi}{\lambda}\right)^{2} \varepsilon_{\mathrm{g}}-\beta^{2}}}\right)+\mathrm{n} \pi,
$$

where $\varepsilon_{\mathrm{g}}$ and $\varepsilon_{\mathrm{c}}$ are the dielectric constants of the guiding and cladding regions of the waveguide, and $\beta$ is the propagation constant of the mode. Here we equate $\lambda$ to the communications wavelength $1.55 \mu \mathrm{m}$ and simplify the comparison between the present designs and that of Yabu et al. by a choice of the channel widths $w_{1}$ and $w_{3}$ close to those in Ref. [3].
Specifically, we set $w_{1}$ and $w_{3}$ equal to 3.0 and $4.0 \mu \mathrm{m}$, respectively, with $\lambda=1.55 \mu \mathrm{m}$, and use Eq. (20) to design the spacing values $d_{1}=3.0 \mu \mathrm{m}$, and the index of refraction values as follows: index of cladding, $n_{\mathrm{o}}$ $=1.444$, index of channels $\mathrm{A}$ and $\mathrm{B}_{1}, n_{1}=1.4589$, index of channels $\mathrm{C}$ and $\mathrm{D}, n_{2}=n_{3}=1.4754$ (with the remaining parameters determined by the symmetry of the structure). The required mismatch between the propagation constants of the modes of channels $B_{1}$ and $\mathrm{C}$ is obtained by the choice of the distinct indices of refraction for the two channels and a design of the width of channel $\mathrm{C}, w_{2}$, equal to $2.5 \mu \mathrm{m} \neq w_{1}$. The value of $w_{1}$, combined with the analysis of Ref. [5], and a choice of $d_{2}$ in Fig. 4, makes possible a computation of the coupling length $\mathrm{L}_{1}$ required to divide the input signal in the gating device equally into the two channels $\mathrm{B}_{1}$ and $\mathrm{B}_{1}{ }^{\prime}$. As an example we choose $d_{2}$ to equal $3.0 \mu \mathrm{m}$, a value consistent with the requirement that the channels $\mathrm{B}_{1}$ and $\mathrm{B}_{1}{ }^{\prime}$ be uncoupled in Region II of Fig. 4. This set of constants determines a value of $\mathrm{L}_{1}$ approximately equal to $468 \mu \mathrm{m}$.

To investigate a potential end-coupling loss in transfer of the symmetric mode of channel $\mathrm{E}$ into the symmetric mode of channel $\mathrm{F}$ we have expanded the field at the exit of channel $\mathrm{E}$ in terms of the single guided mode and the continuum of radiation modes of channel $\mathrm{F}$ and made use of continuity requirements and orthogonality relations to compute the ratio of the power in the guided mode in channel $\mathrm{F}$ to the power in the symmetric mode of channel E. In the absence of reflection at the junction [which previous work (Ref. [5]) has shown to be negligible] we compute this ratio to be 0.97 , consistent with a minimal power loss.

The control of the input signals via phase-matched evanescent field coupling instead of by use of a Y splitter represents an advantage of the geometries of Figs. 1 and 4 over the geometry of Fig. 3. In the design of Fig. 3, to efficiently separate the independent signals at the output of the nonlinear region, the profile of the total field at the output of the nonlinear channel must be made identical to the profile of the total field at the entrance to the channel. This means that the length of the nonlinear region must be a multiple of the coupling length of its two modes, a restriction that is eliminated by the design proposed in Fig. 4 .

The power exchange between the symmetric and antisymmetric modes in the nonlinear region of the gating device in Fig. 4 is illustrated by the graphs of Fig. 5, which show the powers as a function of $z$ in the separate modes of channel $\mathrm{N}$ (per unit width in the $y$ direction) derived from the formula

$$
\mathrm{P}_{n}(z)=\frac{\mathrm{c}^{2}}{8 \pi \omega} \beta_{n}\left|a_{n}(z)\right|^{2},
$$

with the initial powers in the symmetric and antisymmetric modes equated to $38,700 \mathrm{~W} / \mathrm{m}$ and $19,400 \mathrm{~W} / \mathrm{m}$, respectively (consistent with Ref. [3]). The graphs show a periodic transfer of power between the two modes as a function of $z$, with a period 


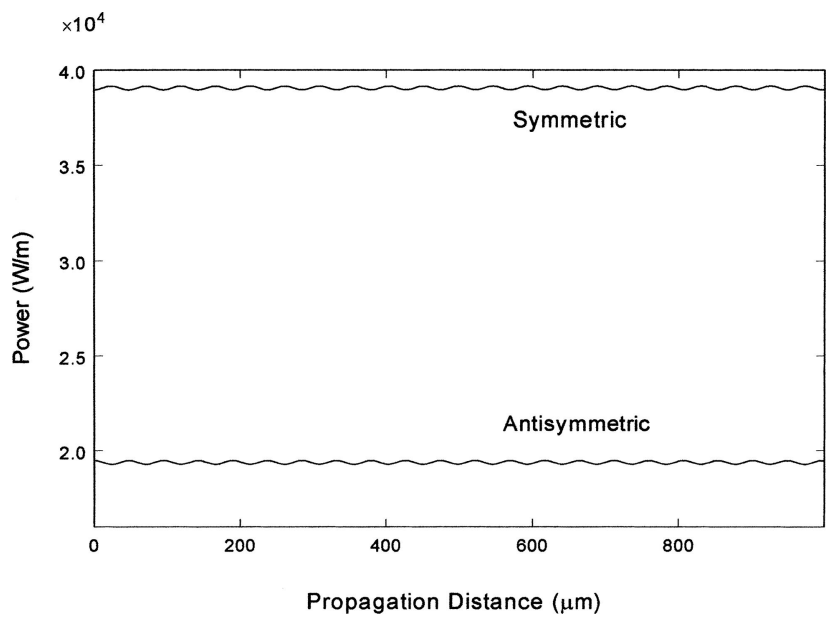

Fig. 5. Graphs of the powers (per unit width in the $y$ direction) in the symmetric and antisymmetric modes of the nonlinear channel $N$ in Fig. 4 as a function of propagation distance $z$, derived from the solution of Eqs. (15). Graphs show power exchanges between the modes resulting from the nonlinearity of the channel.

approximately equal to $45 \mu \mathrm{m}$, which matches the period of the power exchanges in Ref. [3].

The most critical factor in the design of the gate is the $z$-dependent change in relative phase of the antisymmetric (signal) mode produced in the nonlinear medium by the presence of the control field. To determine this phase change, we graph in Fig. 6 the phase $\theta_{2}(z)$ of the antisymmetric mode derived from a numerical solution of Eqs. (15) for the coefficient $a_{2}(z)$, with the coefficient $a_{1}\left(z_{\mathrm{III}}\right)$ at the entrance to the nonlinear medium separately equated to the value at the entrance to channel $\mathrm{C}$ and to zero, respectively. The numerical difference between the two graphs as a function of $z$ is given by the solid line in the same figure and shows that the control signal

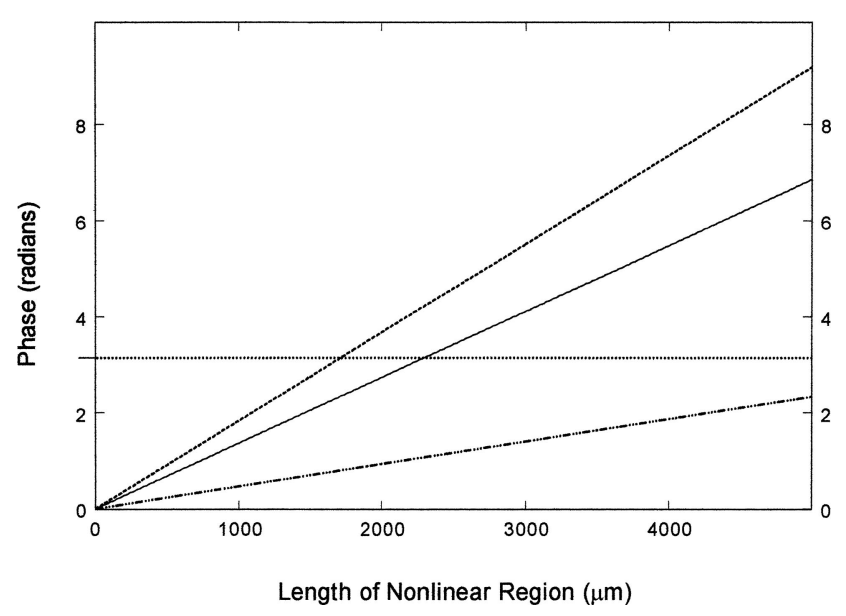

Fig. 6. Graphs of phase $\theta_{2}(z)$ of the antisymmetric mode in channel $N$ in Fig. 4 as a function of $z$ in the separate cases in which the control signal is (a) present (-----) or (b) absent (........). Numerical difference between the graphs is represented by the solid curve that intersects the line corresponding to the phase change of $\pi$ after a propagation distance in the nonlinear channel equal to $2293 \mu \mathrm{m}$.

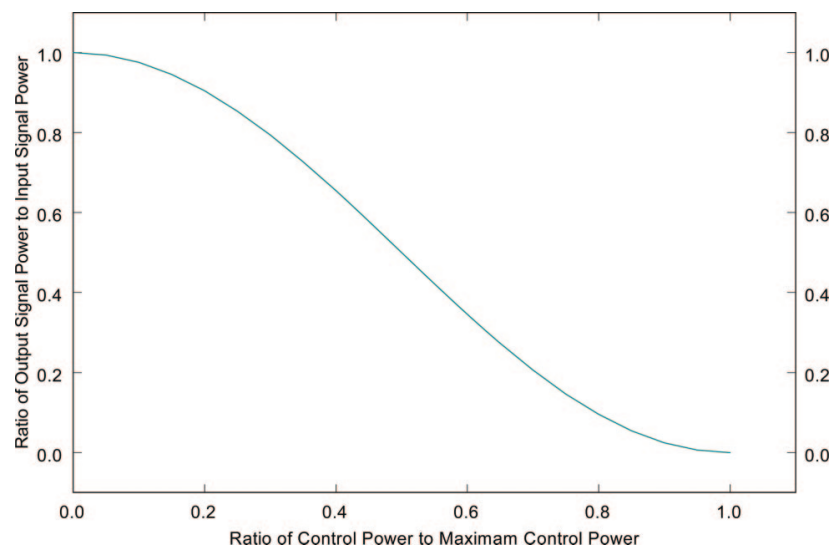

Fig. 7. Graph of output power in channel $X$ in Fig. 4 versus the control power in channel $C$. Both powers are scaled to their maximum values.

changes the relative phase of the antisymmetric mode by $\pi$ during a propagation length in the nonlinear region equal to $2293 \mu \mathrm{m}$. This length equates, by definition, to the length $\mathrm{L}_{\mathrm{III}}$ in Fig. 4 , and its determination completes the selection of design parameters for the gating device. It is a useful feature of the gate that the change in phase of the antisymmetric mode produced by the control beam is an approximately linear function of the length of the nonlinear channel. The use of the computed parameter values in the configuration in Fig. 4, with the power in the control beam equated to $38,700 \mathrm{~W} / \mathrm{m}$, allows the output at port $\mathrm{X}$ to be turned off by the presence of the input signal in channel $\mathrm{C}$, so that the device functions as an all-optical gate. Alternatively, for the same parameters, the variation of the power in channel $\mathrm{C}$ in the range $0-38,700 \mathrm{~W} / \mathrm{m}$, allows the power in the output channel to be varied between zero and its maximum value. The resulting graph of $\mathrm{P}_{\text {out }}$ versus the power in channel $\mathrm{C}$ is shown in Fig. 7 .

The values of the parameters given above make it possible to estimate the size of the device in Fig. 4 by use of the respective formulas for its length and width given by $\mathrm{L}_{\text {Total }}=2\left(\mathrm{~L}_{\mathrm{I}}+\mathrm{L}_{\mathrm{II}}\right)+\mathrm{L}_{\mathrm{III}}$, and $w_{\text {Total }}=$ $2\left(w_{1}+w_{3}+d_{1}+d_{2}\right)+w_{1}$. Using the chosen parameters, we compute $\mathrm{L}_{\text {Total }} \cong 4330 \mu \mathrm{m} \cong 4.3 \mathrm{~mm}$, $w_{\text {Total }}=29 \mu \mathrm{m}$. The compactness of the device here is contributed to by the straight channels of the design in Fig. 4. In contrast, the device in Fig. 3 is expected to be constrained to larger dimensions by the necessity of avoiding bending losses produced by the curved channels of Fig. 3. (Bending losses are minimized in Ref. [3] by the choice of a radius of curvature $R$ equal to $50,000 \lambda$.)

The difference in the lengths of the gating devices in Figs. 3 and 4 can be estimated by comparing the lengths of the corresponding sections of the two devices. The differences arise in the lengths of the coupling regions needed to transfer the signal beams into the nonlinear channels in the two designs. In the design of Yabu et al. [3] the length of the coupling region can be estimated from Fig. 2 of Ref. [3] to be approximately $800 \lambda$, equal to approximately 1240 
$\mu \mathrm{m}$. In comparison, the length of Region II in the design of Fig. 4 is $550 \mu \mathrm{m}$. This difference, doubled in the total device, suggests a length reduction in the present design of approximately $1380 \mu \mathrm{m} \cong$ $1.3 \mathrm{~mm}$, which can be significant if multiple devices are cascaded.

In summary, we propose the use of straight planar waveguides in optical signal combining and processing devices so as to allow for an analytic analysis of these devices that can improve their design and fabrication. In particular we show that their use in an all-optical gate has the potential to both reduce the size of the gate and simplify its fabrication. In addition, the symmetry of the gating device we designed should make it easily cascadable with similar devices in logic circuits for potential applications in optical computing.
M. Wescott acknowledges support from the National Science Foundation Research for Experience for Undergraduate (REU) program under grant 0353899.

\section{References}

1. S. Medhekar and R. K. Sarkar, "All-optical passive transistor," Opt. Lett. 30, 887-889 (2005)

2. Y. Silberberg and P. W. Smith, "Integrated all-optical switching devices," U.S. patent 4,856,860 (15 August 1989).

3. T. Yabu, M. Geshiro, T. Kitamura, K. Nishida, and S. Sawa, "All-optical logic gates containing a two-mode nonlinear waveguide," IEEE J. Quantum Electron. 38, 37-46 (2002).

4. H. Yajima, "Dielectric thin-film optical branching waveguide," Appl. Phys. Lett. 22, 647-649 (1973).

5. M. Mirkov, B. G. Bagley, and R. T. Deck, "Design of multichannel optical splitter without bends," Fiber Integr. Opt. 20, 241254 (2001).

6. Y. Silberberg and G. I. Stegeman, "Nonlinear coupling of waveguide modes,” Appl. Phys. Lett. 50, 801-803 (1987). 